\title{
Zur Benachrichtigung.
}

Die Internationale $\mathscr{H}$ tomgewichtskommission veröffentlicht (soweit möglich) alljährlich die $\mathscr{H}$ tomgewichtszahlen, welche dem jedesmaligen Stande der Wissenschaft entsprechen. Die vorliegende 21. Ruflage der Rechentafeln ist mit den neuesten Zahlen berechnet worden, welche die Internationale Kommission veröffentlicht hat.

R. Thiel. 



\title{
LOGARITHMISCHE RECHENTAPELN PÜR CHEMIKER,
}

\section{PHARMAZEUTEN, MEDIZINER UND PHYSIKER.}

\author{
Begründet von \\ Professor Dr. F. W. Küster †.
}

Für den Gebrauch im Unterrichtslaboratorium und in der Praxis berechnet und mit Erläuterungen versehen.

Nach dem gegenwärtigen Stande der Forschung bearbeitet von

\section{Dr. A. Thiel,}

a. o. Professor der physikalischen Chemie,

Direktor des Physikalisch-chemischen Instituts der C'niveisitát Marburg.

Einundzwanzigste, vermehrte und verbesserte Auflage.

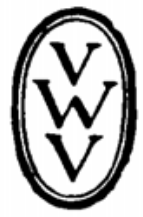

Berlin und Leipzig 1919

Vereinigung wissenschaftlicher Verleger

Walter de Gruyter \& Co.

vorm. G.J. Göschen'sche Verlagshandlung :: J. Guttentag, Verlagsbuchhandlung :: Georg Reimer :: Karl J. Trübner ." Veit \& Comp. 